EESTI NSV TEADUSTE AKADEEMIA TOIMETISED. 31. KOIDE

FUOSIKA * MATEMAATIKA, 1982, NR. 2

ИЗВЕСТИЯ АКАДЕМИИ НАУК ЭСТОНСКОИ ССР. ТОМ 31

ФИЗИКА * МАТЕМАТИКА. 1982, № 2

A. С. СТАРУХИН, К. Н. СОЛОВЬЕВ,

удК 535.37

И. В. СТАНИШЕВСКИИ, А. М. ШУЛЬГА

\title{
ТОНКОСТРУКТУРНЫЕ СПЕКТРЫ ФЛУОРЕСЦЕНЦИИ МЕТАЛЛОКОМПЛЕКСОВ ПОРФИНА ПРИ СЕЛЕКТИВНОМ ЛАЗЕРНОМ ВОЗБУЖДЕНИИ И 4,2 К
}

1. Применение методов молекулярной спектроскопии к исследованию хлорофиллоподобных молекул позволило получить ряд ценных сведений о строении и свойствах таких молекулярных систем. Предложенный относительно недавно метод получения тонкоструктурных спектров многоатомных молекул в произвольных растворителях при селективном возбуждении и $4,2 \mathrm{~K}\left[{ }^{1}\right]$ оказался высокоэффективным при исследовании спектров порфина, его ближайших аналогов и их дейтеропроизводных $\left[{ }^{2}\right]$, а также при получении структурных спектров хлорофилла и его ближайших аналогов.

Исследования тонкоструктурных спектров металлопорфиринов с помощью селективного лазерного возбуждения в системах с сильным неоднородным уширением мы начали с комплексов незамещенного порфина - простейших объектов данного типа. Структура их спектров может служить основой для понимания спектрально-люминесцентных характеристик более сложных биологически активных соединений. В настоящей работе мы уделяем основное внимание двум вопросам: 1) влиянию экстра-лигандов на вибронные спектры металлокомплексов порфина и 2) поляризации индивидуальных бесфононных линий (БФЛ) в спектрах испускания металлопорфиринов. Отметим, что в $\left[{ }^{3,4}\right]$ было отмечено влияние дополнительного комплексообразования на спектры $\mathrm{Mg}$ - и Zn-порфина в матрицах Шпольского. Поляризационным характеристикам спектров поглощения $\mathrm{Pd}$-порфина в монокристаллических матрицах Шпольского (по спектрам возбуждения фосфоресценции) и $\mathrm{Zn-порфина} \mathrm{в} \mathrm{кристаллах} \mathrm{ароматических} \mathrm{углеводородов} \mathrm{посвящены}$ работы $\left[{ }^{5,6}\right]$.

В настоящей работе получены тонкоструктурные вибронные спектры ряда металлокомплексов порфина (Mg-порфина (Mg-П), Zn-пор-

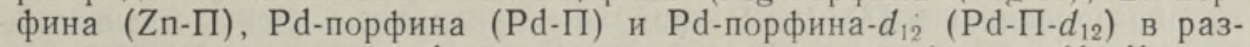
личных растворителях (толуол, толуол-тетрагидрофуран $(1: 1)$, толуол-пиридин $(1: 1)$ и др.) при селективном лазерном возбуждении и $4,2 \mathrm{~K}$.

2. Для исследования тонкоструктурных спектров флуоресценции при селективном возбуждении использовалась установка, собранная на базе спектрометра ДФС-24. Источником монохроматического возбуждения спектров флуоресценции служил перестраиваемый оптический квантовый генератор (ОКГ) с распределенной обратной связью на красителе (родамин-6Ж). Полуширина линии возбуждения составляла $1 \AA$. Накачка ОКГ на красителе осуществлялась второй гармоникой ОКГ ЛТИПЧ-8, который работал с частотой 12,4 Гц. Образцы помещались в оптический гелиевый криостат, где поддерживалась температура жидкого гелия, Методика поляризационных измерений практически иден- 


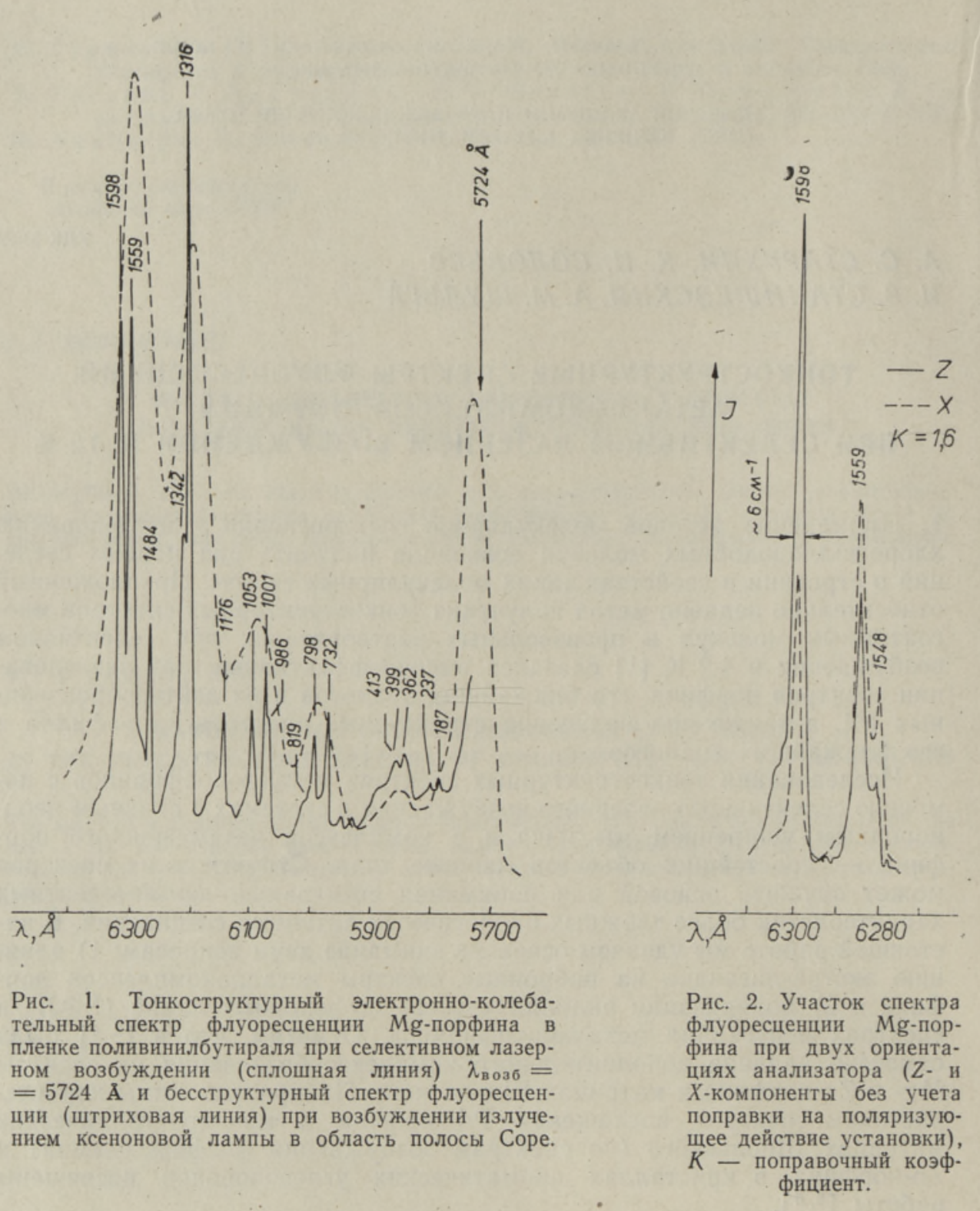

тична описанной в $\left[{ }^{2}\right] . \mathrm{Mg}-П$ вводили в прозрачную пленку поливинилбутираля с концентрацией $10^{-5}$ моль/ $\Omega$ также по методике $\left[{ }^{2}\right]$.

3. Тонкоструктурные спектры флуоресценции всех исследованных соединений были получены при возбуждении в длинноволновый край $0-0$-перехода. На рис. 1 показан спектр флуоресценции $\mathrm{Mg}$-П в пленке поливинилбутираля. Там же пунктирной линией обозначен спектр $\mathrm{Mg}$-П при широкополосном возбуждении в области полосы Соре. Данные о частотах нормальных колебаний, активных в вибронных спектрах перечисленных выше соединений, а также результаты измерений степени поляризации для индивидуальных БФЛ в спектре флуоресценции $\mathrm{Mg}$-П приведены в таблице.

Таблица дополнена значениями частот и симметрией нормальных колебаний $\mathrm{Pd}-П$ из $\left[{ }^{7}\right]$, где исследовались спектры резонансного комбинационного рассеяния (РКР) металлопорфиринов, Қак видно из таб- 
Частоты и симметрия нормальных колебаний металлокомплексов порфина и его дейтеропроизводных

\begin{tabular}{|c|c|c|c|c|c|c|c|c|}
\hline \multicolumn{3}{|c|}{ Pd-порфин } & \multicolumn{2}{|c|}{ Pd-порфин- $d_{12}$} & \multirow{2}{*}{$\begin{array}{c}\begin{array}{c}\text { Zn-пор- } \\
\text { фин }\end{array} \\
\begin{array}{c}v, c \mu^{-1} \\
\text { вибр. }\end{array}\end{array}$} & \multicolumn{3}{|c|}{ Mg-порфин } \\
\hline $\begin{array}{c}v, c M^{-1} \\
\text { вибр. }\end{array}$ & $\begin{array}{c}v, C M^{-1} \\
\mathrm{PKP} \\
{\left[{ }^{7}\right]}\end{array}$ & $\begin{array}{l}\text { симмет- } \\
\text { рия }\end{array}$ & $\begin{array}{l}v, c M^{-1} \\
\text { вибр. }\end{array}$ & $\begin{array}{c}v, c M^{-1} \\
\stackrel{\mathrm{PKP}}{ } \\
{\left[{ }^{7}\right]}\end{array}$ & & $\begin{array}{l}v, c \mu^{-1} \\
\text { вибр. }\end{array}$ & $P, \%$ & $\begin{array}{l}\text { симмет- } \\
\text { рия }\end{array}$ \\
\hline- & 200 & $B_{1 g}$ & - & 200 & 184 & 187 & +15 & $A_{g}$ \\
\hline 270 & - & - & 268 & & - & 237 & - & - \\
\hline $39 \mathrm{C}$ & 390 & $A_{1 g}$ & 381 & 382 & 367 & 362 & +25 & $A_{g}$ \\
\hline 412 & - & - & 390 & - & 408 & 399 & -10 & $B_{1 g}$ \\
\hline 429 & $\overline{720}$ & $\bar{D}$ & 390 & - & 420 & 413 & $\begin{array}{l}-20 \\
+25\end{array}$ & $B_{1 g}$ \\
\hline$[736$ & 732 & $B_{1 g}$ & 662 & 663 & 735 & {$\left[\begin{array}{l}732 \\
739\end{array}\right.$} & $\begin{array}{l}+25 \\
+25\end{array}$ & $\mathrm{Ag}_{\mathrm{g}}$ \\
\hline $\begin{array}{c}{[736} \\
802\end{array}$ & $\begin{array}{l}732 \\
804\end{array}$ & $\begin{array}{l}A_{1 g} \\
A_{2 g}\end{array}$ & $\begin{array}{l}707 \\
761\end{array}$ & 711 & $\begin{array}{c}\text { [735 } \\
799\end{array}$ & $\begin{array}{c}{[732} \\
798\end{array}$ & $\begin{array}{l}+25 \\
-30\end{array}$ & $\begin{array}{l}A_{g} \\
B_{1 g}\end{array}$ \\
\hline 820 & 820 & $\begin{array}{l}A_{2 g} \\
B_{2 g}\end{array}$ & 767 & $\overline{-}$ & 822 & 819 & -20 & $B_{1 g}$ \\
\hline 1018 & 1018 & $A_{1 g}$ & 1012 & 1012 & 997 & 986 & +25 & $A_{g}$ \\
\hline 1033 & $\overline{10 c}$ & - & 1024 & & 1002 & 1001 & +20 & $A_{g}$ \\
\hline 1061 & 1060 & $B_{1 g}$ & 771 & 775 & {$[1058$} & {$[1053$} & +35 & $A_{g}$ \\
\hline- & 1070 & $A_{1 \mathrm{~g}}$ & - & 785 & L1058 & [1053 & +35 & ${ }_{D g}$ \\
\hline- & 1147 & $A_{2 g}$ & - & - & 1150 & 1146 & -25 & $B_{1 \mathrm{~g}}$ \\
\hline$[1194$ & 1193 & $B_{1 g}$ & 969 & 968 & {$[1180$} & {$[1176$} & +40 & $A_{g}$ \\
\hline [1194 & 1193 & $B_{2 g}$ & 1024 & 1028 & L1180 & [1176 & +40 & $A_{g}$ \\
\hline$[1327$ & 1328 & ${ }_{\mathrm{P}}^{A_{2 g}}$ & 1284 & 1284 & {$[1323$} & 1316 & -35 & $B_{1 g}$ \\
\hline$\lfloor 1327$ & $\begin{array}{l}1328 \\
1360\end{array}$ & $\begin{array}{l}B_{2 g} \\
B_{1 g}\end{array}$ & {$\left[\begin{array}{l}1328 \\
1328\end{array}\right.$} & {$\left[\begin{array}{l}1326 \\
1326\end{array}\right.$} & $\begin{array}{r}\mathrm{L} 1323 \\
1360\end{array}$ & $\begin{array}{l}\mathbf{L}_{1316} \\
1355\end{array}$ & $\begin{array}{l}-35 \\
+20\end{array}$ & $\begin{array}{l}B_{1 g} \\
A_{g}\end{array}$ \\
\hline$\left[\begin{array}{l}1358 \\
1358\end{array}\right.$ & 1360 & $\begin{array}{l}D_{1 g} \\
A_{2 g}\end{array}$ & $\begin{array}{r}11328 \\
1198\end{array}$ & $\begin{array}{r}1326 \\
1195\end{array}$ & $\begin{array}{l}1360 \\
1348\end{array}$ & 1342 & -20 & $\begin{array}{l}A_{g} \\
B_{1 g}\end{array}$ \\
\hline- & 1379 & $A_{1 g}$ & - & 1368 & - & - & - & - \\
\hline- & 1440 & $A_{1 g}$ & - & 1421 & - & - & - & - \\
\hline 1498 & 1500 & $B_{1 g}$ & 1448 & 1448 & 1491 & 1484 & +30 & $A_{g}$ \\
\hline 1596 & $\begin{array}{l}1559 \\
1592\end{array}$ & $\begin{array}{l}A_{1 g} \\
A_{2 g}\end{array}$ & $15 \overline{72}$ & $\begin{array}{l}1529 \\
1570\end{array}$ & 1570 & 1559 & $\overline{-}_{-35}$ & $B_{1 g}$ \\
\hline 1631 & 1628 & $B_{18}$ & 1614 & 1612 & 1610 & 1598 & $\begin{array}{l}-20 \\
+20\end{array}$ & $A_{g}$ \\
\hline
\end{tabular}

лицы, наши данные по спектрам флуоресценции $\mathrm{Pd}-\Pi$ и $\mathrm{Pd}-\Pi-d_{12}$ хорошо совпадают. с данными по спектрам РКР [ $\left.{ }^{7}\right]$. Однако по сравнению с результатами работ $\left[{ }^{5,6}\right]$ имеется ряд труднообъяснимых расхождений.

Отметим, что далеко не все колебания, активные в спектрах РКР, активны в спектрах флуоресценции. По результатам $\left[{ }^{7}\right]$ для $\mathrm{Pd}-\Pi, \mathrm{k}$ неактивным в вибронных спектрах можно отнести частоты $1070\left(A_{1 g}\right)$, $1147\left(A_{2 g}\right), 1379\left(A_{1 g}\right), 1440\left(A_{1 g}\right)$ и $1559\left(A_{1 g}\right) c \mu^{-1}$. Следует отметить, что это преимущественно полносимметричные $A_{1 g}$-колебания, что связывается нами с наличием квазизапрета $0-0$-перехода и проявлением колебаний по механизму Герцберга-Теллера. Очевидно, $A_{1 g}$-колебания слабо участвуют в вибронном смешении и неактивны в спектрах. Из полученных спектров были определены также частоты нормальных колебаний, неактивных в спектрах PKР Pd-П: 270, 412, 428 и 1033 cm$^{-1}$.

4. Для выяснения влияния дополнительного комплексообразования нами были получены тонкоструктурные спектры $\mathrm{Mg}-П$ и $\mathrm{Zn}-П$ в чистом толуоле и в толуоле с добавками тетрагидрофурана и пиридина. Влияние экстра-лигандов на тонкоструктурные спектры металлопорфиринов изучим на примере $\mathrm{Mg}$-П. Максимум 0-0-полосы флуоресценции $\mathrm{Mg}-\Pi$ при 4,2 К в толуоле расположен около $5730 \AA$, в смеси толуол-тетрагидрофуран - вблизи $5830 \AA$, а в смеси толуол-пиридин - около $5940 \AA ̊$. . Спектры флуоресценции регистрировались при селективном возбуждении в указанные области. Частоты нормальных колебаний $\mathrm{Mg}$-П при изменении 0-0-перехода вели себя следующим образом:

a) наибольшие изменения испытывали в области $0-500$ cm-1; $^{-1}$; 
б) в области 500-1400 $\mathrm{cm}^{-1}$ практически не менялись при добавках тетрагидрофурана и пиридина;

в) в области $1400-1650 \mathrm{~cm}^{-1}$ понижались до 5-15 $\mathrm{cm}^{-1}$.

Основной причиной этих сдвигов мы считаем ослабление связей $\mathrm{Me}-\mathrm{N}$ при дополнительном комплексообразовании. Кроме того, в различных работах, посвященных тонкоструктурным спектрам порфиринов, применяются неодинаковые среды и добавки, что приводит к различной степени взаимодействия с экстра-лигандом и тем самым к расхождению значений колебательных частот до $10-20 \mathrm{~cm}^{-1}$.

5. Поляризационные измерения выполняли по методике $\left[{ }^{2}\right]$. На рис. 2 соответственно сплошной и пунктирной кривыми показаны интенсивности $Z$ - и $X$-компонент вибронных линий в спектре флуоресценции. Там же дано значение поправочного коэффициента $K$. Измеренные с точностью до $5 \%$ значения степени поляризации $P$ для индивидуальных БФЛ в спектре флуоресценции $\mathrm{Mg}$-П в пленке поливинилбутираля приведены в таблице. Как видно, степень поляризации для колебаний с частотами $399,413,798,819,1146,1316,1342,1559 \mathrm{~cm}^{-1}$ имеет отрицательные значения, изменяющиеся от -10 до $-35 \%$. Положительные ее значения варьируют от +15 до $+40 \%$. Все это говорит о том, что молекулы $\mathrm{Mg}-\Pi$ в поливинилбутирале не обладают симметрией 4-го порядка. Сравнение с аналогичными данными для безметального порфина $\left[{ }^{3,2}\right]$ и с данными спектров PKP Cu-П и $\mathrm{Pd}-\Pi\left[{ }^{7}\right]$ указывает на то, что плоскости симметрии в молекуле Mg-П в данной среде проходят не через метиновые мостики, а через атомы азота пиррольных колец. Наши данные подтвердили отнесение $\left[{ }^{7}\right]$ колебаний металлопорфинов к типам симметрии $B_{1 g}$ и $B_{2 g}$ группы симметрии $D_{4 n}$.

Приведенные результаты показывают эффективность метода тонкоструктурной спектроскопии селективного лазерного возбуждения для исследования внутри- и межмолекулярных взаимодействий в молекулах типа Хл.

Работа выполнена в Центре криогенных исследований АН БССР.

\section{ЛИТ Е Р А Т У Р А}

1. Персонов Р. И., Альшиц Е. А., Быковская Л. А., Письма в ЖЭТФ, 15, вып. 10, 609-612 (1972).

2. Быковская Л. А., Градюшко А. Т., Персонов Р. И., Романов ский Ю. В., Соловьев К. Н., Старухин А. С., Шульга А. М., Ж. прикладной спектроскопии, 29, № 6, 1088-1098 (1978).

3. Гр адюшко А. Т., М ашенков В. А., Соловьев К. Н., Биофизика, 14 , вып. 5 , 827-835 (1969).

4. J a n se n, G., N o o rt, M., Spectrochim. acta, A32, 747-753 (1976).

5. Kielman-van Luijt, E. C. M., Canters, G., J. Mol. Spectrosc., 78, № 3, $469-485$ (1979).

6. B oh a ndy, J., K im, B. F., Spectrochim. acta, A35, 415-420 (1979).

7. Solovyov, K. N., Gladkov, L. L., Gradyushko, A. T., Ksenofontova, N. M., S hulg a, A. M., S tarukhin, A. S., J. Mol. Struct., 45, 267305 (1978).

\section{Институт физики \\ Академии наук Белорусской ССР}


A. S. STARUHHIN, K. N. SOLOVJOV,

I. V. STANISEVSKI, A. M. SULGA

\section{PORFIINI METALLIKOMPLEKSIDE PEENSTRUKTUURSED FLUORESTSENTSISPEKTRID SELEKTIIVSEL LASERERGASTUSEL TEMPERATUURIL $4,2 \mathrm{~K}$}

On saadud Mg-porfiini, Zn-porfiini, Pd-porfiini ja Pd-porfiini- $\mathrm{d}_{12}$ peenstruktuursed elektronvõnkespektrid erinevates orgaanilistes lahustites se!ektiivsel laserergastusel temperatuuril $4,2 \mathrm{~K}$-ja uuritud ekstraligandide mõju $\mathrm{Mg}$ - ja Zn-porfiini normaalvōnkumiste sage: dusele. Mõõdetud on polüvinüülbutüraalis lahustatud Mg-porfiini fluorestsentsispektri individuaalsete foononvabade joonte polarisatsiooniaste. On näidatud, et $\mathrm{Mg}$-porfiini molekulid polümeerses kiles ei oma neljandat järku sümmeetriat ning deformatsiooni suund läbib pürroolirõngaste lämmastikuaatomeid.

A. S. STARUKHIN, K. N. SOLOV'EV,

I. V. STANISHEVSKIJ, A. M. SHUL'GA

\section{FINE-STRUCTURE FLUORESCENCE SPECTRA OF PORPHIN METAL COMPLEXES UNDER A SELECTIVE LASER EXCITATION AT $4.2 \mathrm{~K}$}

Fine-structure vibronic spectra of $\mathrm{Mg}, \mathrm{Zn}, \mathrm{Pd}$ porphins and $\mathrm{Pd}$ porphin- $d_{12}$ in different organic solvents have been obtained under selective laser excitation at $4.2 \mathrm{~K}$. The effect of extra ligands on the frequencies of normal modes active in the f'uorescence spectra of $\mathrm{Mg}$ and $\mathrm{Zn}$ porphins has been investigated (the shifts observed reach $15 \mathrm{~cm}^{-1}$ ). The values of the polarization degree have been measured for individual zero-phonon lines in the fluorescence spectrum of $\mathrm{Mg}$ porphin in polyvinylbutyral. The results are correlated with the data obtained earlier on the resonance Raman spectra of Pd porphin and $\mathrm{Pd}$ porphin- $d_{12}$. It is shown that the $\mathrm{Mg}$ porphin molecules in polymer film do not possess four-fold symmetry, the direction of deformation passing through opposite pyrrole rings. 